\title{
FINITELY DOMINATED COVERING SPACES OF 3- AND 4-MANIFOLDS
}

\author{
JONATHAN A. HILLMAN
}

(Received 1 August 2006; revised 25 October 2007)

Communicated by C. D. Hodgson

\begin{abstract}
If $P$ is a closed 3-manifold the covering space associated to a finitely presentable subgroup $v$ of infinite index in $\pi_{1}(P)$ is finitely dominated if and only if $P$ is aspherical or $\widetilde{P} \simeq S^{2}$. There is a corresponding result in dimension 4, under further hypotheses on $\pi$ and $v$. In particular, if $M$ is a closed 4-manifold, $v$ is an ascendant, $F P_{3}$, finitely-ended subgroup of infinite index in $\pi_{1}(M), \pi$ is virtually torsion free and the associated covering space is finitely dominated then either $M$ is aspherical or $\widetilde{M} \simeq S^{2}$ or $S^{3}$. In the aspherical case such an ascendant subgroup is usually $Z$, a surface group or a $P D_{3}$-group.
\end{abstract}

1991 Mathematics subject classification: 57N13, 20 J05.

Keywords and phrases: finitely dominated, 4-manifold, Poincaré duality group, subnormal subgroup.

A space $X$ is finitely dominated if there is a finite cell complex $Y$ with maps $f: X \rightarrow Y$ and $g: Y \rightarrow X$ such that $g f \sim i d_{X}$. (Thus, $X$ is homotopically a retract of $Y$.) If the universal covering space $\tilde{M}$ of a 4-manifold $M$ is finitely dominated then one of the following holds: $M$ is aspherical; $\tilde{M}$ is homeomorphic to $S^{2} \times R^{2}$ or $S^{3} \times R$; or $\pi=\pi_{1}(M)$ is finite. More generally, if $M$ has a finitely dominated covering space $M_{v}$ such that $v=\pi_{1}\left(M_{v}\right)$ is an $F P_{3}$ normal subgroup of infinite index in $\pi$ there is the additional possibility (when $v$ has infinitely many ends) that $M$ might have a finite covering space which is homotopy equivalent to the mapping torus of a self-homotopy equivalence of a $P D_{3}$-complex. (See $[9$, Theorems 3.9, 10.1 and 11.1].)

In this paper we relax the hypotheses on $v$ further. The arguments we use apply equally well to covering spaces of low-dimensional Poincaré duality complexes. We begin in dimension 3, as the surface case is trivial. In Section 2 we show that if $M$ is a a $P D_{3}$-complex with torsion-free fundamental group $\pi$ and $v$ is a subgroup of infinite index in $\pi$ the associated covering space $M_{v}$ is finitely dominated if and

(C) 2008 Australian Mathematical Society 1446-7887/08 \$A2.00+0.00 
only if $v$ is finitely presentable and $\pi_{2}(M)=0$ or $Z$. In the rest of the paper we consider $\mathrm{PD}_{4}$-complexes. Here we need to assume that the subgroup $v$ be $\mathrm{FP}_{3}$ and ascendant in $\pi$. (The notion of ascendant subgroup is recalled in Section 1 below.) If $M$ is aspherical $\pi$ is a $P D_{4}$-group and finitely dominated covering spaces correspond to $F P_{3}$ subgroups of $\pi$. In Section 3 we show that an ascendant $F P_{3}$ subgroup of infinite index in a $P D_{4}$-group is usually a $P D_{r}$-group for some $r \leq 3$. However, we have not been able to eliminate other possibilities completely. For instance, it is not known whether a Baumslag-Solitar group may be an ascendant subgroup of a $P D_{4}$-group. In Section 4 we consider the case of a $P D_{4}$-complex $M$ with a finitely dominated infinite covering space $M_{v}$ corresponding to an ascendant $F P_{3}$ subgroup $v$, and give homological conditions on $\pi$ and $v$ under which either $M$ is aspherical or $\widetilde{M}$ is homotopy equivalent to $S^{2}$ or $S^{3}$.

\section{Notation}

The Hirsch-Plotkin radical $\sqrt{\pi}$ of a group $\pi$ is the maximal locally nilpotent, normal subgroup of $\pi$. The Hirsch length $h(\nu)$ of a finitely generated nilpotent group $v$ is the number of infinite cyclic factors of a composition series for the group; $h(\sqrt{\pi})$ is the least upper bound of $h(v)$ as $v$ varies over finitely generated subgroups of $\sqrt{\pi}$. If $G$ is a subgroup of $\pi$ then $C_{\pi}(G)$ and $N_{\pi}(G)$ are the centralizer and normalizer of $G$ in $\pi$, respectively. The centre of $G$ is $\zeta G=G \cap C_{\pi}(G)$.

A subgroup $K$ of a group $G$ is ascendant if there is an increasing sequence of subgoups $N_{\alpha}$, indexed by an ordinal $\beth+1$, such that $N_{0}=K, N_{\alpha}$ is normal in $N_{\alpha+1}$ if $\alpha<\beth, N_{\beta}=\cup_{\alpha<\beta} N_{\alpha}$ for all limit ordinals $\beta \leq \beth$ and $N_{\beth}=G$. (If $\beth$ is finite $K$ is subnormal in $G$.) Such ascendant series are well suited to arguments by transfinite induction. For instance, it is easily seen that $\sqrt{K} \leq \sqrt{N}_{\alpha}$, for all $\alpha \leq \beth$. We write $\mathbb{Z}$ for the ring of integers and $Z$ for an abstract infinite cyclic group. If $A$ is an abelian group and $I$ a set let $\oplus^{I} A$ be the direct sum of copies of $A$ indexed by $I$.

We shall assume that the fundamental group $\pi$ of a space or cell complex $X$ acts on the universal cover $\tilde{X}$ on the left, and so the (cellular) chain complex $C_{*}(\widetilde{X})$ is naturally a complex of left $\mathbb{Z}[\pi]$-modules. The equivariant cochain complex $\operatorname{Hom}_{\mathbb{Z}[\pi]}\left(C_{*}(\tilde{X}), \mathbb{Z}[\pi]\right)$ is then a complex of right $\mathbb{Z}[\pi]$-modules. Let $E(\pi)=$ $H^{1}(\pi ; \mathbb{Z}[\pi])$; this is naturally a right $\mathbb{Z}[\pi]$-module.

If $X$ is a Poincaré duality complex with fundamental group $\pi$ and orientation character $w=w_{1}(X)$ and $R$ is a right $\mathbb{Z}[\pi]$-module we let $\bar{R}$ be the conjugate left module, with module structure given by $g . r=w(g) r g^{-1}$ for all $g \in \pi$ and $r \in R$.

\section{2. $P D_{3}$-complexes}

It is easy to see that an infinite covering space of a closed surface is finitely dominated if and only if its fundamental group is finitely generated. Here we show that there is a similar criterion for an infinite covering space of a $P D_{3}$-complex to be finitely dominated. 
LEMMA 1. Let $\pi$ be a finitely generated torsion free group which is not free. Then $E(\pi)$ is a free right $\mathbb{Z}[\pi]$-module.

PROOF. Since $\pi$ is finitely generated it is a free product of finitely many indecomposable groups, and since $\pi$ is torsion-free the latter either have one end or are infinite cyclic. Thus, $\pi$ is an iterated Higman-Neumann-Neumann (HNN) extension with base a nontrivial free product of one-ended groups and trivial associated subgroups. In other words, $\pi$ is the fundamental group of a finite graph of groups $\mathcal{G}$ in which all of the vertex groups have one end and all of the edge groups are trivial. It follows from the Mayer-Vietoris sequences of [1, Theorems 2.10 and 2.11] that $E(\pi)$ is a free right $\mathbb{Z}[\pi]$-module with basis corresponding to the edges of $\mathcal{G}$.

When $\pi$ is a free group $E(\pi)$ is a finitely presentable $\mathbb{Z}[\pi]$-module of projective dimension 1 , and we shall need a different result.

LEMMA 2. Let $\pi=v * \sigma$, where $v$ is finitely generated, and let $I=v \backslash \pi / v$ be the double coset space. Then $\oplus^{I} E(v)$ is a direct summand of the abelian group $E(\pi) \otimes_{v} \mathbb{Z}$.

PROOF. The group $v$ is clearly a retract of $\pi$ and so $H^{1}(\nu ; \mathbb{Z}[\pi])$ is a direct summand of $E(\pi)$ (as a right $\mathbb{Z}[\pi]$-module). Now $H^{1}(\nu ; \mathbb{Z}[\pi]) \cong E(\nu) \otimes_{\nu} \mathbb{Z}[\pi]$, since $v$ is finitely generated. Therefore, $H^{1}(\nu ; \mathbb{Z}[\pi]) \otimes_{\nu} \mathbb{Z} \cong E(\nu) \otimes_{\nu} \mathbb{Z}[\pi / \nu] \cong \oplus^{I} E(\nu)$ is a direct summand of $E(\pi) \otimes_{\nu} \mathbb{Z}$ (as an abelian group).

TheOREM 3. Let $P$ be a $P D_{3}$-complex with fundamental group $\pi$ and let $v$ be a subgroup of infinite index in $\pi$. Then the associated covering space $P_{v}$ is finitely dominated if and only if $\pi$ is virtually $Z$ or $\pi_{2}(P)=0$ and $v$ is finitely presentable.

PROOF. The Hurewicz theorem and Poincaré duality give isomorphisms of left $\mathbb{Z}[\pi]$ modules $\Pi=\pi_{2}(P) \cong H_{2}(P ; \mathbb{Z}[\pi]) \cong \overline{E(\pi)}$. The fundamental group of a $P D_{3}$ complex is a free product of $P D_{3}$-groups with a finitely generated, virtually free group, and so is virtually torsion free [4]. Moreover, a complex is finitely dominated if and only if it has a finite covering space which is finitely dominated. Thus we may assume that $\pi$ is torsion free, after passing to a finite covering space, if necessary.

The spectral sequence of the covering $\widetilde{P} \rightarrow P_{v}$ gives an exact sequence

$$
H_{3}(\nu ; \mathbb{Z}) \rightarrow \mathbb{Z} \otimes_{\nu} \Pi \rightarrow H_{2}\left(P_{\nu} ; \mathbb{Z}\right) \rightarrow H_{2}(\nu ; \mathbb{Z}) \rightarrow 0 .
$$

We may assume that $v$ is finitely presentable. It is then a finite free product of finitely presentable subgroups of $P D_{3}$-groups with a free group, by the Kurosh subgroup theorem. In particular, $H_{s}(v ; \mathbb{Z})$ is finitely generated for all $s \geq 0$, and so $H_{2}\left(P_{v} ; \mathbb{Z}\right)$ is finitely generated if and only if $\mathbb{Z} \otimes_{v} \Pi$ is finitely generated.

If $\pi$ is free of rank 1 then $\pi \cong Z$ and $E(\pi) \cong \mathbb{Z}$. Hence, $\pi_{2}(P)$ is infinite cyclic, so $\widetilde{P} \simeq S^{2}$. In this case every covering space is finitely dominated. 
If $\pi$ is free of rank $r>1$ then we may assume that $\nu$ is a proper free factor of $\pi$, after passing to a subgroup of finite index, if necessary [3]. We may also assume that $P$ is orientable. It is easy to see that the double set space $I=v \backslash \pi / v$ is infinite. Since $\mathbb{Z} \otimes_{\nu} \Pi \cong \overline{E(\pi) \otimes_{\nu} \mathbb{Z}}$ is not finitely generated, by Lemma $2, P_{v}$ cannot be finitely dominated.

If $\pi$ is not free $\Pi \cong \mathbb{Z}[\pi]^{s}$ for some $s \geq 0$, by Lemma 1 . Thus, $\mathbb{Z} \otimes_{\nu} \Pi$ is free of infinite rank as an abelian group, unless $s=0$. Thus, if $P_{v}$ is finitely dominated $s=0$ and so $\Pi=0$.

Suppose conversely that $\Pi=0$ and that $v$ is a finitely presentable subgroup of infinite index in $\pi$. Then the universal covering space $\widetilde{P}$ is contractible, and so $P$ is aspherical. Therefore $c . d . v \leq 2$, by [16], and $P_{v} \simeq K(v, 1)$. Let $Y$ be the finite 2-complex determined by a finite presentation for $\nu$. The cellular chain complex for $\tilde{Y}$ gives an exact sequence of $\mathbb{Z}[v]$-modules

$$
0 \rightarrow \pi_{2}(Y) \rightarrow C_{2} \rightarrow C_{1} \rightarrow C_{0} \rightarrow \mathbb{Z} \rightarrow 0
$$

where $C_{q}=C_{q}(\tilde{Y})$ is a finitely generated free $\mathbb{Z}[v]$-module, for $q \leq 2$. Since $c . d . v \leq 2$ the module of 1 -cycles $Z_{1}=\operatorname{Im}\left(\partial_{2}\right)$ is projective, and so $C_{2} \cong Z_{1} \oplus \pi_{2}(Y)$. Thus, $\pi_{2}(Y)$ is a finitely generated projective module. Let $F$ be a free $\mathbb{Z}[v]$-module of countably infinite rank. Then $\pi_{2}(Y) \oplus F \cong F$, by the 'Eilenberg swindle'. Hence, we may construct a $K(\pi, 1)$ complex $K$ by adding countably many 3-cells to $Y \vee V$, where $V$ is a countable wedge of 2-spheres. Let $c: Y \rightarrow K$ be the classifying map and $p: K \rightarrow Y$ be the map which collapses $V$ and the adjoined 3-cells. Then $c p \sim i d_{K}$, and so $P_{v} \simeq K$ is finitely dominated.

In particular, a closed 3-manifold has a finitely dominated infinite covering space if and only if its universal covering space is contractible or homotopy equivalent to $S^{2}$ or $S^{3}$.

\section{Poincaré duality groups}

Subgroups of $P D_{n}$-groups are the algebraic analogues of covering spaces of aspherical $P D_{n}$-complexes. The analogues of finitely dominated covering spaces are the $F P_{n-1}$ subgroups, for which the trivial module $\mathbb{Z}$ has a projective resolution which is finitely generated in degrees $\leq n-1$. (There is then a finite projective resolution of length at most $n$, since either $v$ is a $P D_{n}$-group or $c . d . v<n$ [16].) The algebraic notion is broader in one respect: we do not assume that the $P D_{n}$-groups or their $F P$ subgroups are finitely presentable.

In [10] it was shown that if $v$ is an $F P_{2}$ ascendant subgroup of infinite index in a $P D_{3}$-group $\pi$ then either $v \cong Z$ and is normal in $\pi$ or $v$ is a $P D_{2}$-group and $\left[\pi: N_{\pi}(\nu)\right]<\infty$ or $\pi$ is a virtually poly- $Z$ group (and every subgroup is $F P_{2}$ ).

THEOREM 4. Let $G$ be a nontrivial $F P_{3}$ normal subgroup of infinite index in a $P D_{4}$ group $\pi$. Then either: 
(1) $G$ is a $P D_{3}$-group and $\pi / G$ has two ends;

(2) $G$ is a $P D_{2}$-group and $\pi / G$ is virtually a $P D_{2}$-group; or

(3) $G \cong Z, H^{s}(\pi / G ; \mathbb{Z}[\pi / G])=0$ for $s \leq 2$ and $H^{3}(\pi / G ; \mathbb{Z}[\pi / G]) \cong Z$.

Proof. The subgroup $G$ is $F P$, since $c . d . G<4$ (see [16]), and hence so is $\pi / G$. The $E_{2}$ terms of the Lyndon-Hochschild-Serre (LHS) spectral sequence with coefficients $\mathbb{Q}[\pi]$ can then be expressed as tensor products $E_{2}^{p q}=H^{p}(\pi / G ; \mathbb{Q}[\pi / G]) \otimes$ $H^{q}(G ; \mathbb{Q}[G])$. If $H^{j}(\pi / G ; \mathbb{Q}[\pi / G])$ and $H^{k}(G ; \mathbb{Q}[G])$ are the first nonzero such cohomology groups then $E_{2}^{j k}$ persists to $E_{\infty}$. Hence, $j+k=4$ and $E_{2}^{j k} \cong \mathbb{Q}$. Therefore, $H^{j}(\pi / G ; \mathbb{Q}[\pi / G])$ and $H^{n-j}(G ; \mathbb{Q}[G])$ each have dimension 1 over $\mathbb{Q}$. In particular, $\pi / G$ has one or two ends and $G$ is a $P D_{4-j}$-group over $\mathbb{Q}[6]$. If $\pi / G$ has two ends then it is virtually $Z$, and so $G$ is a $P D_{3}$-group (over $\mathbb{Z}$ ), by [1, Theorem 9.11]. If $H^{2}(G ; \mathbb{Q}[G]) \cong H^{2}(\pi / G ; \mathbb{Q}[\pi / G]) \cong \mathbb{Q}$ then $G$ and $\pi / G$ are virtually $P D_{2}$-groups [2]. Since $G$ is torsion-free it must be a $P D_{2}$-group. The only remaining possibility is (3).

Do the conclusions of this theorem hold if the hypothesis that $G$ be $F P_{3}$ is relaxed to ' $G$ is $F P_{2}$ '? (If $G$ is an $F P_{2}$ normal subgroup of a $P D_{4}$-group $\pi$ and $\pi / G$ is virtually a $P D_{r}$-group then $G$ is a $P D_{4-r}$-group [11].) If $v . c . d . \pi / G<\infty$ then $\pi / G$ is virtually a $P D$-group in case (3) also, by [1, Theorem 9.11].

COROLlary. If $G$ is an $F_{3} P_{3}$ normal subgroup of infinite index in $\pi$ and $K$ is an ascendant $F P_{2}$ subgroup of $G$ then $K$ is a $P D_{k}$-group for some $k<4$.

PROOF. This follows immediately from Theorem 4 together with the [10, corollary of Theorem 11].

We shall consider next $F P_{3}$ ascendant subgroups of $P D_{4}$-groups.

THEOREM 5. Let $G$ be a nontrivial $F P_{3}$ ascendant subgroup of infinite index in a $P D_{4}$-group $\pi$. If $G$ has finitely many ends then one of the following holds:

(1) $G$ is a $P D_{3}$-group, $\left[\pi: N_{\pi}(G)\right]<\infty$ and $N_{\pi}(G) / G$ has two ends;

(2) c.d.G $=3$ and $H^{2}(G ; \mathbb{Z}[G])$ is not finitely generated as an abelian group;

(3) $G$ is a $P D_{2}$-group, $\left[\pi: N_{\pi}(G)\right]<\infty$ and $\pi$ is virtually the group of a surface bundle over a surface;

(4) $G$ is a $P D_{2}$-group, $\zeta G=1$ and $\pi$ is virtually the group of the mapping torus of a self homeomorphism of a surface bundle over the circle;

(5) $\quad$ c.d.G $=2, \quad \chi(G)=0, H^{2}(G ; \mathbb{Z}[G])$ is not finitely generated as an abelian group and $\left[\pi: N_{\pi}(G)\right]=\infty ;$ or

(6) $\quad G \cong Z$ and either $G<\sqrt{\pi}<\pi$ is a subnormal chain or $\pi$ is virtually nilpotent of Hirsch length 4.

PROOF. Let $G=N_{0}<N_{1}<\cdots<N_{\beth}=\pi$ be an ascendant sequence, and let $\phi$ be the union of the finite ordinals $\leq \beth$. If $G$ is normal in $\pi$ then the theorem follows from Theorem 4. Otherwise, replacing $N_{1}$ by the union of the terms $N_{\alpha}$ which normalize $G$ and reindexing, if necessary, we may assume that $G$ is not normal in $N_{2}$. 
Since $[\pi: G]=\infty$ we have $c . d . G<4$, by [16]. Suppose first that $c . d . G=3$ and that $H^{2}(G ; \mathbb{Z}[G])$ is finitely generated as an abelian group. Then $H^{s}(G ; \mathbb{Z}[G])=0$ for $s \leq 2$, by [5] or [2]. If $\phi$ is infinite then $N_{\phi}$ is not finitely generated, and so $c . d . N_{\phi}=4$, by [8, Theorem 3.3]. However, then [ $\left.\pi: N_{\phi}\right]<\infty[16]$ and so $N_{\phi}$ is finitely generated. Therefore, $\phi$ is finite, so $N_{\phi}$ is one-ended, $F P$ and ascendant in $\pi$, and it is easily seen that the theorem holds for $G$ if it holds for $N_{\phi}$. Thus, we may assume that $\left[N_{1}: G\right]=\infty$. It follows immediately from the LHS spectral sequence that $H^{s}\left(N_{1} ; W\right)=0$ for $s \leq 3$ and any free $\mathbb{Z}\left[N_{1}\right]$-module $W$. Hence, c.d. $N_{1}=4$ and so $\left[\pi: N_{1}\right]<\infty$, by [16]. Hence, $N_{1}$ is a $P D_{4}$-group and (1) follows from Theorem 4. If c.d.G $=3$ and $H^{2}(G ; \mathbb{Z}[G])$ is not finitely generated as an abelian group (2) holds.

Suppose now that c.d.G $=2$ and that $\chi(G) \neq 0$. If $\left[N_{i}: G\right]$ is finite, then $\chi(G)=$ $\left[N_{i}: G\right] \chi\left(N_{i}\right)$. Hence, we again find that $\phi$ is finite. If $G_{1}<G_{2}$ are two such groups with $G_{1}$ normal in $G_{2}$, then $\left[G_{2}: G_{1}\right]$ is finite, by [1, Theorem 8.2]. Moreover, if $G_{2}$ is normal in $J$ then $\left[J: N_{J}\left(G_{1}\right)\right]<\infty$, since $G_{2}$ has only finitely many subgroups of index $\left[G_{2}: G_{1}\right]$. Therefore, we may assume that $G$ is maximal among normal subgroups of $N_{1}$ with cohomological dimension 2 and that $\left[N_{1}: G\right]=\infty$. If $N_{1}=\pi$, then (3) holds, by Theorem 4. Otherwise, we may assume that $G$ is not normal in $N_{2}$, as observed earlier, and so there is an $n$ in $N_{2}$ such that $n G n^{-1} \neq G$. Let $H=$ $G . n G n^{-1}$. Then $G<H$ and $H$ is normal in $N_{1}$, so $[H: G]=\infty$ and c.d. $Q H=3$. Moreover, $H$ is $F P$ and $H^{s}(H ; \mathbb{Z}[H])=0$ for $s \leq 2$, so either $N_{1} / H$ is locally finite or $c . d_{\mathbb{Q}} N_{1}>c . d . \mathbb{Q} H$, by [1, Theorem 8.2]. If $N_{1} / H$ is locally finite but not finite, then we again have $c . d_{\mathbb{Q}} N_{1}>c . d \cdot \mathbb{Q} H$, by [8, Theorem 3.3]. If $c . d \cdot{ }_{\mathbb{Q}} N_{1}=4$, then $\left[\pi: N_{1}\right]<\infty$, so $N_{1}$ is a $P D_{4}$-group and (3) holds, by Theorem 4. Otherwise $\left[N_{1}: H\right]<\infty$ and then c.d. $N_{1}=3, N_{1}$ is $F P$ and $H^{s}\left(N_{1} ; \mathbb{Z}\left[N_{1}\right]\right)=0$ for $s \leq 2$. Hence, $N_{1}$ is a $P D_{3}$-group by (1), and so (4) holds.

Suppose that $\chi(G)=0$ and that $G$ is a $P D_{2}$-group. Then $G \cong Z^{2}$ or $Z \times_{-1} Z$, so $h(\sqrt{\pi}) \geq 2$ and $\chi(\pi)=0$. We may assume that $\pi$ is orientable, so $\operatorname{Hom}(\pi, Z) \neq 0$. If $h(\sqrt{\pi})>2$ then $\pi$ is virtually poly-Z, by [9, Theorem 8.1]. Therefore, we may also assume that $h(\sqrt{\pi})=2$. In this case $\sqrt{\pi} \cong Z^{2}$ and $\pi$ is virtually the group of a torus bundle over a surface, by $[9$, Theorem 9.2]. Since $[\sqrt{\pi}: G]<\infty$ it follows also that $\left[\pi: N_{\pi}(G)\right]<\infty$ and so (3) holds. If $c . d . G=2$ but $G$ is not a $P D_{2}$-group then $H^{2}(G ; \mathbb{Z}[G])$ is not finitely generated as an abelian group [6] and $\left[\pi: N_{\pi}(G)\right]=\infty$, and so (5) covers the remaining possibilities with one end.

If $G$ has two ends, then $G \cong Z$, so $G \leq \sqrt{\pi}$. If $h=h(\sqrt{\pi}) \leq 2$ then $\sqrt{\pi}$ is abelian of rank $h$, by [9, Theorem 9.2]. If $h>2$ then $\pi$ is virtually poly- $Z$ of Hirsch length 4 , by [9, Theorem 8.1]. If $\sqrt{\pi}$ is abelian or nilpotent of class 2 then $G$ is a normal subgroup of $\sqrt{\pi}$; otherwise $\pi$ is virtually nilpotent of type $\mathbb{N} i l^{4}$, by [9, Theorem 1.5].

To what extent can the hypotheses be relaxed? Are all ascendant $F P$ subgroups $P D$-groups? If so then cases (2) and (5) cannot arise. (This is certainly so if there is a subnormal sequence consisting of $F P$ subgroups.) Can a finitely generated noncyclic free group be an ascendant subgroup of a $P D_{4}$-group? 
EXAmple. Let $G$ be a $P D_{2}$-group such that $\zeta G=1$. Let $\theta: G \rightarrow G$ have infinite order in $\operatorname{Out}(G)$, and let $\lambda: G \rightarrow Z$ be an epimorphism. Let $\pi=(G \times Z) \times{ }_{\phi} Z$ where $\phi(g, n)=(\theta(g), \lambda(g)+n)$ for all $g \in G$ and $n \in Z$. Then $G$ is subnormal in $\pi$ but this group is not virtually the group of a surface bundle over a surface.

Any group with a finite two-dimensional Eilenberg-Mac Lane complex is the fundamental group of a compact aspherical 4-manifold with boundary, obtained by attaching 1- and 2-handles to $D^{4}$. On applying the reflection group trick of Davis to the boundary we see that each such group embeds in a $P D_{4}$-group (see [12]). The simplest such groups $G$ with $\chi(G)=0$ which are not $P D_{2}$-groups are the Baumslag-Solitar 1-relator groups $G_{p, q}=\left\langle a, t \mid t a^{p} t^{-1}=a^{q}\right\rangle$ with $|p q|>1$. Can they be realized as ascendant subgroups of $P D_{4}$-groups?

\section{4. $P D_{4}$-complexes}

In this section we consider $P D_{4}$-complexes $M$ with a finitely dominated covering space associated to an ascendant $F P_{3}$ subgroup of $\pi_{1}(M)$.

TheOREM 6. Let $M$ be a P D $D_{4}$-complex with fundamental group $\pi$ and let $v$ be an ascendant $\mathrm{FP}_{3}$ subgroup of infinite index in $\pi$. Suppose that the associated covering space $M_{v}$ is finitely dominated. Then:

(1) if $v$ is finite then the universal covering space $\tilde{M}$ is contractible or homotopy equivalent to $S^{2}$ or to $S^{3}$, and $\left[\pi: N_{\pi}(v)\right]$ is finite;

(2) if $v$ has one end then $M$ is aspherical;

(3) if $v$ has two ends then either $M$ is aspherical or it is finitely covered by $S^{2} \times S^{1} \times S^{1}$ or $h(\sqrt{\pi})=1$ and $H^{2}(\pi ; \mathbb{Z}[\pi])$ is not finitely generated as an abelian group;

(4) if $v$ has infinitely many ends and $v \leq N$ where $N$ is an $F P_{2}$ normal subgroup of infinite index in $\pi$ then either $M$ has a finite covering space which is homotopy equivalent to the mapping torus of a self-homotopy equivalence of a $\mathrm{PD}_{3}$ complex and $\left[\pi: N_{\pi}(\nu)\right]$ is finite or $M$ is aspherical and $N$ is not $F P_{3}$.

PROOF. Let $v=N_{0}<N_{1}<\cdots<N_{\beth}=\pi$ be an ascendant sequence. Suppose first that $v$ is finite. Then $\widetilde{M}$ is also finitely dominated, and so is contractible (in which case $v=1$ ) or is homotopy equivalent to $S^{2}$ or $S^{3}$, by [9, Theorem 3.9]. If $\widetilde{M} \simeq S^{2}$ the kernel of the natural homomorphism from $\pi$ to $\operatorname{Aut}\left(\pi_{2}(M)\right)$ is torsion free. Hence, $v=Z / 2 Z$ and so $v$ is central in $N_{1}$. Moreover as it is the torsion subgroup of $\zeta N_{1}$ it is characteristic in $N_{1}$, and hence normal in $N_{2}$. Transfinite induction now shows that $\nu$ is normal in $\pi$. If $\widetilde{M} \simeq S^{3}$ then $\pi$ has two ends, and so $\left[\pi: N_{\pi}(\nu)\right]$ is finite.

If $v$ is infinite then transfinite induction using the LHS spectral sequence, [8, Theorem 3.3] and [15, Lemma 4.1] shows that $\pi$ has one end, and that if $v$ has one end $H^{2}(\pi ; \mathbb{Z}[\pi])=0$. Since $v$ is $F P_{3}$ and $M_{v}$ is finitely dominated $\pi_{2}(M)=$ $\pi_{2}\left(M_{\nu}\right)$ is finitely generated as a $\mathbb{Z}[\nu]$-module, and so $\operatorname{Hom}_{\pi}\left(\pi_{2}(M), \mathbb{Z}[\pi]\right)=0$. 
Therefore, $\pi_{2}(M) \cong \overline{H^{2}(\pi ; \mathbb{Z}[\pi])}$, by [9, Lemma 3.3]. In particular, if $v$ has one end then $\pi_{2}(M)=0$ and so $M$ is aspherical.

If $v$ has two ends then it has an infinite cyclic normal subgroup of finite index, and so we may assume without loss of generality that $v \cong Z$. Hence $v \leq \sqrt{\pi}$. If $h(\sqrt{\pi})>2$ then $H^{2}(\pi ; \mathbb{Z}[\pi])=0$, by [9, Theorem 1.16], and so $M$ is aspherical. (In fact $M$ is then homeomorphic to an infrasolvmanifold, by [9, Theorem 8.1].) If $h(\sqrt{\pi})=2$ and $\sqrt{\pi}$ has infinite index in $\pi$, then we again have $H^{2}(\pi ; \mathbb{Z}[\pi])=0$ and so $M$ is aspherical. (If $\sqrt{\pi}$ is finitely generated it is nilpotent, hence $F P$, and the vanishing of $H^{2}(\pi ; \mathbb{Z}[\pi])$ follows immediately from an LHS spectral sequence argument. If $\sqrt{\pi}$ is not finitely generated then it is the increasing union of finitely generated subgroups of Hirsch rank 2, and we may apply [8, Theorem 3.3] to conclude that $H^{s}(\sqrt{\pi} ; \mathbb{Z}[\pi])=0$ for $s \leq 2$.) If $h(\sqrt{\pi})=2$ and $\sqrt{\pi}$ has finite index in $\pi$ then $\pi$ is virtually $Z^{2}$. We may then assume that $\pi \cong Z^{2}$ and $\pi / v \cong Z$. Since $H_{*}\left(M_{\nu} ; \mathbb{Q}\right)$ is finitely generated it follows from the Wang sequence for the projection of $M_{v}$ onto $M$ that $\chi(M)=0$. Hence, $M$ is finitely covered by $S^{2} \times S^{1} \times S^{1}$, by [9, Theorem 10.10].

Suppose that $h(\sqrt{\pi})=1$ and let $\sqrt{M}$ be the associated covering space. Since $h(v)=h(\sqrt{\pi})$ the stages of a subnormal chain between $v$ and $\sqrt{\pi}$ are locally finite, and so the rational homology spectral sequences between the corresponding covering spaces collapse, to show that $H_{*}(\sqrt{M} ; \mathbb{Q})$ is finitely generated and $\chi(\sqrt{M})=\chi\left(M_{v}\right)$. In particular, $\pi / \sqrt{\pi}$ has finitely many ends, since $H_{3}(\sqrt{M} ; \mathbb{Q})$ is finite dimensional.

If $[\pi: \sqrt{\pi}]$ is finite then $\sqrt{\pi}$ is finitely generated. However, then $[\sqrt{\pi}: \nu]<\infty$ and so $[\pi: \nu]<\infty$, contrary to hypothesis.

If $\pi / \sqrt{\pi}$ has two ends then we may assume that $\pi / \sqrt{\pi} \cong Z$. However, then $\pi$ is an ascending HNN construction over a finitely generated base, and so the torsion subgroup $T$ of $\sqrt{\pi}$ is finite, while $\sqrt{\pi} / T$ is abelian. Therefore, $\sqrt{\pi}$ has a finitely generated infinite normal subgroup and so $H^{2}(\pi ; \mathbb{Z}[\pi])$ is free abelian [13]. Since $H_{*}(\sqrt{M} ; \mathbb{Q})$ is finitely generated $\sqrt{M}$ satisfies Poincaré duality with simple coefficients $\mathbb{Q}$ and formal dimension 3 [14] and so $\chi(\sqrt{M})=0$. Hence $\chi\left(M_{v}\right)=$ 0 . This in turn implies that $\pi_{2}\left(M_{\nu}\right)$ is a torsion $\mathbb{Z}[v]$-module. Now $\pi_{2}\left(M_{\nu}\right)$ is finitely generated as a $\mathbb{Z}[v]$-module, and is $\mathbb{Z}$-torsion-free, since $\pi_{2}\left(M_{\nu}\right)=\pi_{2}(M) \cong$ $H^{2}(\pi ; \mathbb{Z}[\pi])$. Therefore, $\pi_{2}\left(M_{\nu}\right)$ is finitely generated as an abelian group, since $\mathbb{Z}[v] \cong \mathbb{Z}\left[t, t^{-1}\right]$. Since $\pi$ has elements of infinite order $H^{2}(\pi ; \mathbb{Z}[\pi])$ must therefore be 0 or $Z$, by [5, Corollary 5.2]. But $M$ cannot be aspherical as c.d. $(\pi) \leq$ c.d. $\mathbb{Q} \sqrt{\pi}+c . d . \mathbb{Q} Z=2$. Therefore, $\tilde{M} \simeq S^{2}$. As $\pi$ is elementary amenable it must be virtually $Z^{2}$, by [9, Theorem 10.10]. However, this contradicts the assumption that $h(\sqrt{\pi})=1$. Therefore, $\pi / \sqrt{\pi}$ has one end. As we may again exclude the possibility that $H^{2}(\pi ; \mathbb{Z}[\pi]) \cong Z$, either $M$ is aspherical or $H^{2}(\pi ; \mathbb{Z}[\pi])$ is not finitely generated as an abelian group.

Suppose that $v$ has infinitely many ends and $v \leq N$ where $N$ is an $F P_{2}$ normal subgroup of infinite index in $\pi$. If $[N: v]$ is finite then $N$ has infinitely many ends and $M_{N}$ is finitely dominated, so $\pi / N$ has two ends and the covering space associated to $N$ is a $P D_{3}$-complex, by [9, Theorem 3.9]. If $[N: v]=\infty$ then $N$ has one end (as above). 
Hence $H^{s}(\pi ; \mathbb{Z}[\pi])=0$ for $s \leq 2$ and so $M$ is aspherical, as before. This cannot happen if $N$ is $\mathrm{FP}_{3}$, by the corollary to Theorem 4.

The hypothesis that $v$ be $F P_{3}$ is used to ensure that $\operatorname{Hom}_{\pi}\left(\pi_{2}(M), \mathbb{Z}[\pi]\right)=0$, and is automatic if $\pi$ is finite or has two ends. Does the theorem hold without this hypothesis?

Products $M=S^{1} \times N$ where $N=S^{3}, S^{2} \times S^{1},\left(S^{1}\right)^{3}$ or $\left(S^{2} \times S^{1}\right) \#\left(S^{2} \times S^{1}\right)$ give examples realizing most of the possibilities allowed by the theorem. The main exception is the final alternative in case (3); the following corollary suggests that this is rather unlikely.

COROLLARY. If $v$ has finitely many ends and either $\sqrt{\pi}$ is abelian or $h(\sqrt{\pi}) \neq 1$ then $M$ is aspherical or $\widetilde{M}$ is homotopy equivalent to $S^{2}$ or $S^{3}$.

PROOF. We may assume that $\sqrt{\pi}$ is abelian of rank 1 and $\pi / \sqrt{\pi}$ has one end. However, then $H^{2}(\pi ; \mathbb{Z}[\pi])=0$, by [7] and [13], and so $M$ is aspherical.

In case (4) the question raised after Theorem 4 also remains: is every $F P_{2}$ normal subgroup of a $\mathrm{PD}_{4}$-group $\mathrm{FP}_{3}$ ?

What happens if we drop the hypothesis on ascendancy? If a $P D_{4}$-complex $M$ has a finitely dominated infinite covering space must $\pi_{1}(M)$ have one or two ends?

\section{References}

[1] R. Bieri, Homological dimensions of discrete groups, Queen Mary College Mathematics Notes (Queen Mary College, London, 1976).

[2] B. H. Bowditch, 'Planar groups and the Seifert conjecture', J. Reine Angew. Math. 576 (2004), $11-62$.

[3] R. G. Burns, 'A note on free groups', Proc. Amer. Math. Soc. 23 (1969), 14-17.

[4] J. Crisp, 'The decomposition of 3-dimensional Poincaré duality complexes', Comment. Math. Helv. 75 (2000), 232-246.

[5] F. T. Farrell, 'The second cohomology group of $G$ with coefficients $Z / 2 Z[G]$ ', Topology 13 (1974), 313-326.

[6] F. T. Farrell, 'Poincaré duality and groups of type $F P^{\prime}$ ', Comment. Math. Helv. 50 (1975), 187-195.

[7] R. Geoghegan and M. L. Mihalik, 'A note on the vanishing of $H^{n}(G ; Z[G])$ ', J. Pure Appl. Algebra 39 (1986), 301-304.

[8] D. Gildenhuys and R. Strebel, 'On the cohomological dimension of soluble groups', Canad. Math. Bull. 24 (1981), 385-392.

[9] J. A. Hillman, Four-manifolds, geometries and knots, Geometry and Topology Monographs, 5 (Geometry and Topology Publications, University of Warwick, Coventry, 2002).

[10] J. A. Hillman, 'Centralizers and normalizers in $P D_{3}$-groups and open $P D_{3}$-groups', J. Pure Appl. Algebra 204 (2006), 244-257.

[11] J. A. Hillman and D. S. Kochloukova, 'Finiteness conditions and $P D_{r}$-covers of $P D_{n}$-complexes', Math. Z. 256 (2007), 45-56.

[12] G. Mess, 'Examples of Poincaré duality groups', Proc. Amer. Math. Soc. 110 (1990), 1144-1145.

[13] M. L. Mihalik, 'Ends of double extension groups', Topology 25 (1986), 45-53.

[14] J. W. Milnor, 'Infinite cyclic coverings', Conference on the Topology of Manifolds (ed. J. G. Hocking) (Prindle, Weber and Schmidt, Boston, London, Sydney, 1968), pp. 115-133. 
[15] D. J. S. Robinson, 'On the cohomology of soluble groups of finite rank', J. Pure Appl. Algebra 6 (1975), 155-164.

[16] R. Strebel, 'A remark on subgroups of infinite index in Poincaré duality groups', Comment. Math. Helv. 52 (1977), 317-324.

JONATHAN A. HILLMAN, School of Mathematics and Statistics, University of Sydney, NSW 2006, Australia

e-mail: jonh@maths.usyd.edu.au 